\title{
Modelling of Liquid Flow Control System Using Optimized Genetic Algorithm
}

\author{
Pijush Dutta ${ }^{1, *}$, Asok Kumar ${ }^{2}$ \\ ${ }^{1}$ Department of Electronics and Communication Engineering, Global Institute of Management and Technology, Nadia, India \\ ${ }^{2}$ Department of Student Welfare, Vidyasagar University, Midnapore, India
}

\begin{abstract}
Estimation of a highly accurate model for liquid flow process industry and control of the liquid flow rate from experimental data is an important task for engineers due to its non linear characteristics. Efficient optimization techniques are essential to accomplish this task.In most of the process control industry flow rate depends upon a multiple number of parameters like sensor output,pipe diameter, liquid conductivity ,liquid viscosity, liquid density etc. In traditional optimization technique its very time consuming for manually control the parameters to obtain the optimal flow rate from the process. Hence the alternative approach , computational optimization process is utilized by using the different computational intelligence technique.In this paper three different selection of Genetic Algorithm is proposed to taste against the present liquid flow process. The proposed algorithm is developed based on the mimic genetic evolution of species that allow the consecutive generations in population to adopt their environment. Equations for Response Surface Methodology (RSM) and Analysis of Variance (ANOVA) are being used as non-linear models and these models are optimized using the proposed different selection of Genetic optimization techniques. It can be observed that the among these three different selection of Genetic Algorithm ,Rank selected GA is better than the other two selection (Tournament ,Roulette wheel) in terms of the accuracy of final solutions, success rate, convergence speed, and stability.
\end{abstract}

Keywords Liquid flow control process; Anemometer type flow sensor; Modelling;Genetic Algorithm;Analysis of Variance (ANOVA);Response Surface Methodology (RSM)

AMS 2010 subject classifications 13P25, 97R40

DOI: $10.19139 /$ soic-2310-5070-618

\section{Introduction}

In most of the process control industry, there is a no requirement to calculate the input to a process that will steer its outputs in a craved way and thus attain some optimum (desired) goal. Most of the process control system is threatened due to illegitimate input parameter settings $[1,2]$.To optimize the performance of a multivariable process control system through the classical method is obstinate and gradual.The main snag of the classical optimization is to getting the retaliation influenced by individual independent variables than other input variables should be kept constant thats inreciprocative between the input variables are absent.Precautional provision, the total number experimental trials are increased which is time elapsing. Thats why alternative approach is taken where mathematical modelling (computational optimization) of the process is designed (inputCoutput relationship) using various computational intelligence techniques. The model could be based on either physical phenomena or the historical inputCoutput data for a provisioned system. Once the model is developed, mathematical techniques can be applied to determine the inputs to the process that will persuade a certain given criterion.A number of

\footnotetext{
${ }^{*}$ Correspondence to: Pijush Dutta (Email: pijushdutta009@gmail.com). Department of Electronics and Communication Engineering,
} Global Institute of Management and Technology ,Nadia, India (741102).

ISSN 2310-5070 (online) ISSN 2311-004X (print)

Copyright (C) 2020 International Academic Press 
literature survey is conduct on the different types of optimization techniques applied in flow rate control system. A semiconductor based Anemometer type flow meter is designed in [3], and [4] propose to eliminate the non linearity relation between the flow rate \& Anemometer flow sensor output. Bera et al.[5] shows a comparative study between the matched pair transistors flow meter and platinum resistance temperature detector. The results showed a linear relationship between the sensor output and flow rate; whereas for the turbulent flow the relationship followed a non linear relation.Santhosh KV and BK Roy [6] proposed a model to make the quantification system adaptive to variations in pipe diameter, liquid density, and liquid temperature using optimized using ANN.Proposed measurement technique attains the objectives quite decently. Therefore,estimation of a highly accurate model for describing a liquid flow control process is still an open problem to us.

[7] designed an intelligent fuzzy logic model for ultrasonic flow sensor to determine the flow rate in for known experimental inputs pipe diameter, liquid density \& temperature. This model not only produce a full scale linearity between sensor output \& flow rate but the minimum rmse error adjusted by the model is upto $7.72 \%$. Application of intelligent fuzzy logic controller proposed by [11],to predict the flow rate in anemometer flow sensor in liquid flow process system they applied the different no \& different nature of membership function to optimized the flow rate. From the experimental result analysis it is seen that least rmse error is 7.98\% [13] used ANN for prediction of liquid flow rate passing through the anemometer sensor in a liquid flow process .A feed forward neural network model was developed exploiting experimental measurements. The neural network model was trained and tested using MATLAB toolbox .The results predicted from ANN model is compared with experimental measurements. Investigation shows that the maximum rmse error is $2.94 \%$ for for learngdm adaptive learning function \& trainlm training function with a good correlation. [10] proposed SVM and KNN algorithm to classify data and get prediction (find hidden patterns) for target. Here they use nominal data to classify in liquid flow process control system and discover the data pattern to predict future data sets.

[11] investigated a Hybrid GA-ANN model is developed \& employed for the prediction \& optimization of liquid parameter, Anemometer sensor output \& pipe diameter.From the numerical result it is observed that among the three different selection rank selected hybrid Genetic Algorithm- Neural network (GA-ANN) model is better than the other two selections Tournament \& Roulette wheel with accuracy $98.42 \%$ of final solutions. [12] examined anemometer thermal sensor based process model for optimizing the flow rate .Results is in good agreement with the experimental results \& can be applied to predict the performance of mass flow sensor. For the best ANFIS structure RMSE and MAE were calculated as $2.143 \%$ \& $0.504 \%$ respectively.

[13] also designed a model of liquid flow processes using an artificial neural network (NN) and optimizes it using a flower pollination algorithm (FPA) to improve the accuracy and convergence speed. In the first phase, the NN model was trained by the dataset obtained from the experiments, $\&$ model response was cross-verified with the experimental results and found to be satisfactory. In the second phase of work minimum flow rate was found for the optimized conditions of sensor output voltages, pipe diameter and liquid conductivity. Accuracy after cross-validation and testing sub datasets was nearly $94.17 \%$ and $99.25 \%$, respectively.[14] proposed three different improved versions of original Elephant Swarm Water Search Algorithm (ESWSA) and tested against the present problem of liquid flow control. Equations for Response Surface Methodology (RSM) and Analysis of Variance (ANOVA) are being used as non-linear models and these models are optimized using those newly proposed optimization techniques. Results show that these proposed improved ESWSA metaheuristics have superior performance over original ESWSA and can predict the liquid flow rate with more accuracy, efficiency and reliability.

Metaheuristics [15] are the one of the most popular subclass optimization techniques where optimization processes are typically inspired by physical phenomena, animals behaviors, or evolutionary concepts. clarity,resilience, derivation-free mechanism and local optima evasion capability make metaheuristics highly germane for real-life optimization problems. Most popular Swarm Intelligence (SI) techniques are as follows: Particle swarm optimization (PSO)[16],Bat Algorithm (BA)[17] ,Cuckoo Search(CS) [18] ,Flower 
Pollination algorithm (FPA) [19],Firefly Algorithm (FA)[20],Ant Colony Optimization (ACO)[21] ,Artificial Bee Colony(ABC)[22] ,Grey Wolf Optimization [23], Whale Optimization Algorithm [24] etc. However, according No Free Lunch theorem [25],there is no single metaheuristics which is proper for solving all kinds of optimization problems.Therefore, suggesting new metaheuristics and variation of current approaches(with good convergence speed, good accuracy, less computational time, less number of parameters to be tuned, good exploration and exploitation capability) is still very enticing field of study to solve a real-life optimization problem like modelling of liquid flow control process .

Genetic algorithms (GA) approach based on natural biological evolution motivated by the Darwinian Principle of evolution through genetic selection .Genetic Algorithm is a robust, globally applied optimization technique compared to traditional optimization paradigms. It can be used not only for general optimization problems, but also in different optimization and unconventional optimization problems. So far basic GA have been applied on many problems in different disciplines successfully, such are parameter optimization in parallel hybrid electric vehicles [26],calculate yield \& cost of the solar hot water system [27], In energy saving building system [28],optimization model of a linear laminated elastic plate [29], data mining [30], pattern recognition [31] and optimization of Hybrid Rockets where both the continuous \& discrete variables [32].

The main objective of this work is to propose or develop some efficient optimization techniques such that we can model (computationally) the liquid flow control process accurately and reliably. In this study, three different parent selected version of Genetic Algorithm evolutionary algorithm have been proposed (by introducing good diversity $\&$ population in a few generation ). These parent selected GA are tested against the problem of modeling of liquid flow control process. Here, response surface methods (RSM) and analysis of variance (ANOVA) are used as the mathematical models for modeling of liquid flow control process. The details of the ANOVA and RSM based are discussed in next section. The rest of this paper is organized as follows: after introduction, flow sensor design \& operation described in section 2 and the experimental set up, mathematical model \& genetic algorithm is briefly introduced in Section 3 . Section 4 described the results \& discussion and finally conclusions are presented in Section 5 .

\section{Flow sensor Design}

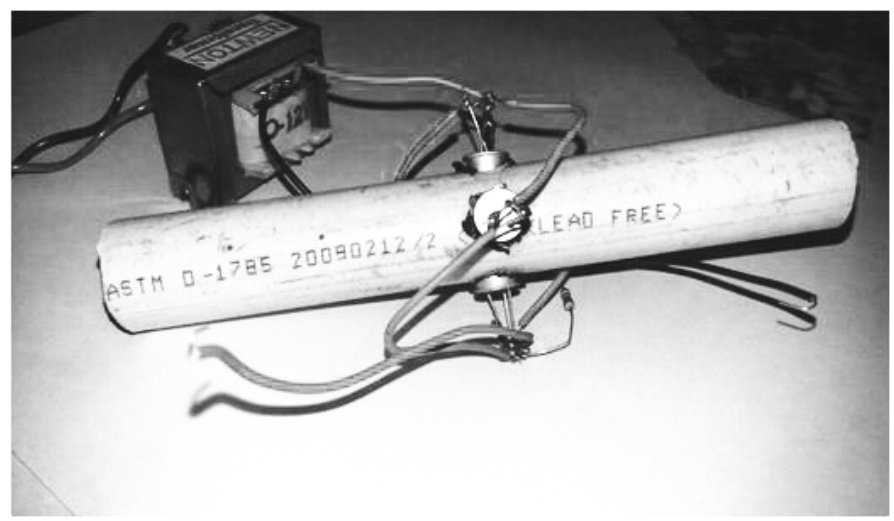

Figure 1. Semiconductor based Anemometer

Figure 1 represents the semiconductor based Anemometer Flow Sensor. Present research done by the semiconductor based Anemometer flow sensor instead of the other type of flowsensor which are used in process control industry alternatives of present work sensors are electromagneic flow sensor, Ultrasonic flow sensor,Hall effect flow sensor,Venturi meter, Ultrasonic flow sensor etc. This sensor has the following advantage :low cost, apply 
the cooling technique, Doppler effect, fluid suction pump and energy association are neglected,can be applicable for wide range of fluid speed (upto $600 \mathrm{lpm}$ for the present experiment)by means of convection method,long time research tool and provides high resolution and less interference of noise on output.Anemometer flow sensor designed by placing four transistor in diametrical plane of a pvc pipe at right angles to each other to form a bridge circuit. Base and emitter terminal of each transistor are shorted to form $P$ terminal while collector terminal consider as $N$ terminal so that transistor can be consider as conventional $P N$ junction diode.After forming a wheatstone bridge circuit one pair of transistor operating in a forward biased mode while the opposite arm transistor operating in a reverse bias.Due to the cooling technique the change in resistance for the forward biased transistor and reverse biased transistor will be different.The resulting bridge output voltage is sum of the positive and negative half cycle output voltage which are again linearly depends on the change in forward biased resistance. As the change in resistance is linearly propotional to the flow rate .Hence sensor output produce a linear voltage corresponding to the flow rate.

\section{Experimental setup for the liquid flow process}

The experimental work is carried out with the Flow and level measurement and control set up [13].The set up is used along with the flowing parts which are given in Table1.

Table 1. Experimental Setup

\begin{tabular}{|l|l|}
\hline Machine/tools & Specification/Description \\
\hline $\begin{array}{l}\text { process control setup Flow \& Level } \\
\text { measurement and Control }\end{array}$ & Model no. WFT -20-I \\
\hline Anemometer Flow sensor & Designed by the SL 100 transistor \\
\hline PVC pipe & Diameter with 20mm,25mm \& 30mm \\
\hline Digital Multimeter & $3 \frac{1}{2}$ \\
\hline Rota meter & $\begin{array}{l}\text { Taking the reading of the Flow rate } \\
\text { ranging 0-600 lpm }\end{array}$ \\
\hline
\end{tabular}

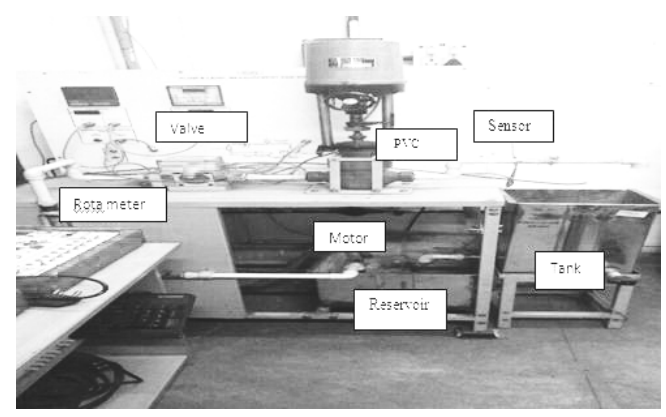

Figure 2. Experimental set up for liquid flow rate measurement [13]

The experimental work is done in a process control setup Flow and Level measurement and Control ( model no. WFT -20-I) shown in Figure 2 In the present investigation, the liquid velocities measured were in the range 0lpm $-6001 \mathrm{pm}$. Flow sensor voltages were calibrated against Liquid flow velocities which was determined by a special mass flow control unit, to an inaccuracy of 1 percent from the reading. Overall temperature variation of the liquid was typically less than $0.5 \mathrm{C}$ during the course of the entire experiment at room temperature. The purpose of water Flow control process is to keep the water flow in the tube at a desired rate and track the reference trajectory. In this paper water is considered as the liquid to check the non-linearity of the cylindrical tank. Reservoir tank collects the water which is pumped to the cylindrical tank. Flow is calculated by using anemometer type flow sensor. In 
this experimental set up water is pumped up in ploy vinyl chloride (PVC)pipe from reservoir tank. A DC motor is connected in reservoir to drive the system the rate of change of the water flow is measured in Rota meter indicator. Non linear electrical signal is achieved across the non contact type liquid flow sensor connected at the end of the PVC pipe. Here we use Transistor based Flow sensor where four transistor connected in a diametrical plane of the PVC pipe to form a Bridge type full wave rectifier. Change in water flow affects the output of the sensor signal. Water from the sensor is fall into the cylindrical tank which is again connected to the main water reservoir through a pipe so that cyclic process is formed. pneumatic control valve allows water flow into the tube from the tank and causes flow rate change in the tube. The operation is repeated throughout the control process till the water flow rate in the tube is set to reference. A reference trajectory or flow rate is first set to be followed by the system. From the above experimental setup we get sensor output voltage with respect to the variation of the water flow rate under the different combination of pipe diameter and water parameters [13].Experiments are carried out at different flow rates ,sensor output ,pipe diameter and liquid density.The output variable is considered as liquid flow rate predicted by the optimization technique defined by the function of input parameter sensor output ,pipe diameter and liquid density.The experimental conditions are shown in Table 2.

Table 2. Ranges of the process parameters

\begin{tabular}{|c|c|}
\hline Process Conditions (Input parameters) & Range of the parameters \\
\hline Sensor output voltage & $210 \mathrm{mv}$ to $285 \mathrm{mv}$ \\
\hline Pipe diameter(mm) & $20 \mathrm{~mm}, 25 \mathrm{~mm} \& 30 \mathrm{~mm}$ \\
\hline Water conductivity $(\mathrm{W} / \mathrm{m} . \mathrm{k})$ & $606,615 \& 622(\mathrm{~W} / \mathrm{m} . \mathrm{k})$ \\
\hline Water Viscosity & $725.4,779.7 \& 898.2($ pas.sec$)$ \\
\hline Water Viscosity & $725.4,779.7 \& 898.2($ pas.sec $)$ \\
\hline Water Density & $993.9,995.6 \& 996.9(\mathrm{~kg} / \mathrm{m} 3)$ \\
\hline
\end{tabular}

For this work, total 134 sample data has been observed which consist of four independent variables sensor output voltage , pipe diameter, liquid (water) conductivity \& viscosity .Among these 134 datasets 17 number of datasets are used for the testing purpose shown in table3. To conduct this research, we had taken the 3 different set of pipe diameter i.e. $20 \mathrm{~mm}, 25 \mathrm{~mm}$ and $30 \mathrm{~mm}$. For each of the cases we collect data of the flow rate as a experimental output data for different sensor output voltage, pipe diameter ,liquid conductivity \& viscosity .liquid density is assumed to be constant as overall temperature variation of the liquid was typically less than $0.5 \mathrm{C}$ during the course of the entire experiment at room temperature. Experimental data are shown in Table 3.

\subsection{Mathematical Description of the Problem}

Due to the non linear characteristics of the semiconductor based Anemometer flow sensor we get a variation of sensor output voltage with the change in liquid flow rate by considering the different values of the experimental pipe diameter where the sensor is placed in a diametrical plane [4]. Again sensor output voltage depends on the pipe diameter ,liquid water viscosity \& conductivity \& partially depends upon the liquid density which is ignored when we construct the fitness function of flowrate.So, any change in pipe diameter it is very difficult to recalibrate the conventional controller circuit each time to predict the output and it is also a time consuming process.To overcome such type of drawbacks (i.e. manual recalibration) we need to develop a mathematical model using some computational intelligence tools which may help us to establish a relationship between liquid flow rate,sensor voltage and pipe diameter, experimental liquid (water ) conductivity \& viscosity. These models will also help to find out the optimal operating condition and to predict the flow rate under certain condition (i.e. for particular values of diameter, sensor output voltage, water viscosity \& conductivity) without recalibration. However, in this work, we have considered equations of two widely popular statistical methods namely Response Surface Methodology (RSM) [33], \& [34] and Analysis of Variance (ANOVA) [35] as nonlinear models to describe the relationship between variables of liquid flow control process.RSM is a collection of mathematical and statistical techniques, generally used for empirical model building and analyzing a problem. Response surface methodology is one of most extensive application mathematical tool for a situations where several input variables potentially influence 
Table 3. Experimental datasets for liquid flow control process

\begin{tabular}{|c|c|c|c|c|}
\hline Sensor output & Diameter & Conductivity & Viscosity & Flow rate \\
\hline 0.218 & 0.024 & 0.606 & 0.8982 & 0.0008 \\
0.221 & 0.025 & 0.616 & 0.7797 & 0.0008 \\
0.225 & 0.025 & 0.616 & 0.8982 & 0.0016 \\
0.232 & 0.025 & 0.597 & 0.7797 & 0.0016 \\
0.234 & 0.020 & 0.616 & 0.8982 & 0.0024 \\
0.237 & 0.027 & 0.622 & 0.7797 & 0.0024 \\
0.238 & 0.030 & 0.606 & 0.7254 & 0.0024 \\
0.239 & 0.025 & 0.616 & 0.8982 & 0.0032 \\
0.241 & 0.027 & 0.622 & 0.7797 & 0.0032 \\
0.245 & 0.024 & 0.606 & 0.7254 & 0.0032 \\
0.247 & 0.024 & 0.616 & 0.8982 & 0.0040 \\
0.247 & 0.025 & 0.622 & 0.7797 & 0.0040 \\
0.250 & 0.025 & 0.606 & 0.7254 & 0.0048 \\
0.256 & 0.025 & 0.616 & 0.8982 & 0.0048 \\
0.254 & 0.024 & 0.622 & 0.7797 & 0.0056 \\
0.259 & 0.030 & 0.606 & 0.7254 & 0.0064 \\
0.265 & 0.027 & 0.622 & 0.7797 & 0.0072 \\
\hline
\end{tabular}

some performance measure or quality characteristics (response) of the process. RSM used higher order polynomial equation to describe the relationships amongst variables. The second order models are flexible and can take on wide variety of functional forms. Therefore, in this present problem, the liquid flow rate (F) can be expressed in terms of sensor output (E),pipe diameter (D), conductivity (k) \& viscosity(n) using RSM as follows:

$$
\begin{array}{r}
F=\beta_{0}+\beta_{1} E+\beta_{2} D+\beta_{3} k+\beta n+\beta_{11} E^{2}+\beta_{22} D^{2}+\beta_{33} k^{2}+\beta_{44} n^{2}+\beta_{12} E D \\
+\beta_{13} E k+\beta_{14} E n+\beta_{23} D k+\beta_{24} D n+\beta_{34} k n
\end{array}
$$

where $\beta_{0}, \beta_{0}, \beta_{1}, \beta_{2}, \beta_{3}, \beta_{4}, \beta_{11}, \beta_{22}, \beta_{33}, \beta_{44}, \beta_{12}, \beta_{13}, \beta_{14}, \beta_{23}, \beta_{24} \& \beta_{34}$ are the regression coefficients. Values of the coefficients are needed to be estimated using some computational intelligence techniques from the experimental dataset. ANOVA [34] is used to test for significant differences among sample without assuming any parametric relationships. In ANOVA, the relationship between the variables can be expressed in term of non-linear power equations. In this present problem, the liquid flow rate $(F)$ can be expressed in term of sensor output $(E)$, pipe diameter $(D)$, conductivity $(k) \&$ viscosity (n)using ANOVA as follows:

$$
F=\mu_{1} E_{2}^{\mu} D_{3}^{\mu} k_{4}^{\mu} n_{5}^{\mu}
$$

where $\mu_{1}, \mu_{2}, \mu_{3}, \mu_{4}, \& \mu_{5}$ are the coefficients for this model, whose values are needed to be estimated using some computational intelligence techniques from the experimental dataset.

\subsection{Optimization of the mathematical model}

Finding out the values of coefficients of nonlinear model in Eqs. 1 and Eqs. 2 of liquid flow rate is essentially a nonlinear optimization process. Normally, metaheuristics are used to fit the calculated characteristic of liquid flow control process to the experimental one. The estimation task aims to seek the most optimal values for the unknown parameters so as to minimize the error between the measured and simulated flow rate. The root mean square of the error $\operatorname{RMSE}(\mathrm{X})$ is defined as Eqs. 3 can be used as the objective function for the metaheuristic which is used for modelling of liquid flow control process. eqn where $N$ is the number of the experimental data, $X$ is the set of the estimated parameters. For RSM based modelling, the error function $\left(E_{i}, D_{i}, k_{i}, n_{i}, X\right)$ and set of parameters $X$ 
can be written as

$$
\begin{array}{r}
f\left(E_{i}, D_{i}, k_{i}, n_{i}, X\right)=\beta_{0}+\beta_{1} E+\beta_{2} D+\beta_{3} k+\beta n+\beta_{11} E^{2}+\beta_{22} D^{2}+\beta_{33} k^{2}+\beta_{44} n^{2}+\beta_{12} E D \\
+\beta_{13} E k+\beta_{14} E n+\beta_{23} D k+\beta_{24} D n+\beta_{34} k n-F
\end{array}
$$

$\mathrm{X}=\beta_{0}, \beta_{0}, \beta_{1}, \beta_{2}, \beta_{3}, \beta_{4}, \beta_{11}, \beta_{22}, \beta_{33}, \beta_{44}, \beta_{12}, \beta_{13}, \beta_{14}, \beta_{23}, \beta_{24} \& \beta_{34}$

For ANOVA based modelling, the error function $\left(E_{i}, D_{i}, k_{i}, n_{i}, X\right)$ and set of parameters $\mathrm{X}$ can be expressed in Eqs. 4:

$$
f\left(E_{i}, D_{i}, k_{i}, n_{i}, X\right)=\mu_{1} E_{2}^{\mu} D_{3}^{\mu} k_{4}^{\mu} n_{5}^{\mu}-F
$$

$\mathrm{X}=\mu_{1}, \mu_{2}, \mu_{3}, \mu_{4}, \& \mu_{5}$

Obviously, smaller objective function value gives better solution which corresponds to superior set of estimated model parameters or coefficients

\subsection{Proposed Methodology}

In this research work, we have used basic Genetic Algorithm optimization (GA) and its three versions Roulette wheel ,Tournament \& Random process for optimization or modelling of flow measurement problem. Therefore, initially basic Genetic algorithm(GA) was elaborated and the characteristics of the improved version GA are elaborated later accordingly.

3.3.1. Basic Genetic Algorithm Genetic algorithms (GAs) are a subclass of what are known as evolutionary algorithms [36]. These are computational models that mimic natural evolution in their design and implementation; i.e. they are based on survival of the fittest. GAs differ from conventional search techniques in that they operate on a coded parameter set of the solution, are global in their search, make use of a cost function that does not involve derivatives and finally employ pseudo-probabilistic rules and not deterministic ones. Genetic algorithms have been used in recent years in solving optimization problems in science and engineering applications [37], [38], [39] and [30]. Implementation of GAs involves making the following preliminary decisions.

1. Solution encoding. This involves coding a possible solution (individual) as a string of variables using some alphabet, e.g. binary 0,1 . Individuals are likened to chromosomes and variables to genes. A chromosome (solution) is composed of several genes (variables).

2. Evaluation function. This determines the fitness score attached to each chromosome (solution). The higher this score, the greater is the chance of an individual (solution) being selected for reproduction.

3. Fitness :fitness function is the computational process which evaluate the quality of a chromosome that would be the solution of a particular problem. In analogy chromosome referred as a genotype can be convert into a solution (phenotypes)of a problem is very complicated process, where the fitness function goes through a number of criteria and objectives such as completetion time, cost minimization ,resource utilization etc.

4. Selection criterion. In selection process the best individual is selected as a parents so that it can contribute population for the next generation.

5. Recombination/reproduction. This is achieved through two genetic operators, namely crossover and mutation. A number of variations of crossover are in use such as single-point, multi-point or uniform crossover. In single-point crossover where binary encoding is used, a locus (bit location) is randomly chosen. Bits after that locus are exchanged between two chromosomes to create two offspring (new solutions). Mutation on the other hand involves randomly flipping some of the bits in a string (chromosome). A very small probability is usually attached to occurrence of mutation at each bit location (e.g. 0.001). This operation is performed to ensure that new areas of the solution are explored.

6. Termination criteria. The algorithm can be terminated if the maximum number of generations (iterations) is achieved, or convergence of the solution is attained (i.e. all solutions yield the same fitness value or differ by less than a specified tolerance). 


\section{Results and Discussion}

To verify its performance of the proposed Three different selection of Genetic Algorithm, the algorithms are tested on parameters or coefficients estimation problems for modelling of liquid flow control process. Here, the algorithms are tested against both cases of RSM or ANOVA based model as described earlier section where 117 number of data sets are used to train the data \& construct the objective function of liquid Flow rate using ANOVA \& RSM . The experimental dataset has been obtained from the laboratory experimentation as mentioned in earlier section. The dataset consists of 17 data points of sensor output voltage $(E)$, pipe diameter $(D)$ and liquid flow rate $(\mathrm{F})$ liquid conductivity $(\mathrm{k})$ \& liquid viscosity $(n)$.This experimental dataset has been used a test dataset for the parametric optimization of Genetic Algorithm based model of liquid flow control process. Objective functions for these two cases are RMSE which are already discussed in earlier section. After optimization, best set of coefficients can be obtained. In this section, we present the numerical simulation results of GA and Three different selection of Genetic Algorithm on liquid flow control process problem. Moreover, we also perform comparison among them and give statistical analysis of the evaluated results. For all algorithms, population and maximum iteration number are set to 100 and 5000 respectively. For RSM based model, search space is restricted to12 i.e. we have considered 12 dimensional function optimization problems to search optimal values of the coefficients $\left\{\beta_{0}, \beta_{1}, \beta_{2}, \beta_{3}, \beta_{4}, \beta_{11}, \beta_{22}, \beta_{33}, \beta_{44}, \beta_{12}, \beta_{13}, \beta_{14}, \beta_{23}, \beta_{24} \& \beta_{34}\right\}$. For ANOVA based model, the search space is limited to 5 i.e. we have considered 5 dimensional function optimization problems in search of optimal values of coefficients $\mu_{1}, \mu_{2}, \mu_{3}, \mu_{4}$, and $\mu_{5}$. The search range for the optimization of liquid flow control process model is set to $[-75,75]$ for all of the coefficients for both types of modelling. All the techniques were simulated using Matlab 2015b in a computer with 4 GB RAM, Intel (R) core (TM) i3 processor and Windows7 operating System. Due to stochastic nature of metaheuristics, they may give different output depending different random initialization. Therefore, each algorithm is executed for 20 times for each cases and the statistical analysis has been carried out from the obtained simulated results. During these numerical experimentations, we have tested and compared the efficiency of the proposed algorithm on the basis of some criterions such fitness test, reliability test, computational efficiency test, convergence test and accuracy test which are described in following subsection one by one. At the end, overall performances have been discussed. For implementation of GA following Parameters settings is considered for optimizing the input variables shown in Table 4.

Table 4. Parameter Setting for GA modeling of flow rate

\begin{tabular}{|c|c|}
\hline Parameter of Genetic Algorithm & Value \\
\hline No of decision variables to be optimized & 5 \\
\hline Search range for the optimization & -75 to 75 \\
\hline No of Iteration & 100 to 5000 \\
\hline Population size & 100 \\
\hline Cross over percentage & 0.7 \\
\hline Extra range factor for crossover & 0.4 \\
\hline Mutation rate & 0.1 \\
\hline Length of the chromosome & $6 \mathrm{bit}$ \\
\hline
\end{tabular}

\subsection{Fitness Test}

Final output or fitness value of an optimization algorithm is the most important criterion to prove its efficiency. Using above mentioned parameters setting, we optimized benchmark function by using three different selection method in Genetic Algorithm in both the cases of ANOVA \& RSM method. Here, we have considered three important criterions (output) namely worst (maximum) fitness, best (minimum) fitness and mean (average) fitness values which are obtained after 20 times program run. A metaheuristic should give smaller values of maximum, minimum and mean of fitness as much $R M S E$ as possible for better performances. In this work, the fitness is calculated from the rmse of the algorithms. Comparative studies based on these criterions are shown in Table 5. 
From this Table, it can be seen that proposed Roulette wheel selection can reach best fitness value (minimum rmse i.e.3.03E-05) for ANOVA based modelling which are shown in bold letters; whereas Roulette wheel \& Rank selection methods in Genetic algorithms are able to reach each best fitness value (minimum rmse i.e. 5.41E-05) for RSM based modelling. For worst fitness Roulette wheel based selection GA is able to achieve lowest worst fitness value for ANOVA based modelling; whereas all the there selection methods (Roulette wheel ,Tournament \& Rank ) are able to achieve to smallest value of worst fitness i.e. maximum rmse for RSM based modelling. Mean RMSE is found by the Roulette wheel selection which is the best result for ANOVA based modelling. On the other hand Roulette wheel \& Rank selection has superior in term of mean of rmse for RSM based modelling. It is interesting to observe that minimum rmse for ANOVA based modelling of liquid flow process (obtained by all algorithms) is far better than minimum rmse for RMSE based modelling. It indicates that ANOVA based modelling performs much better than RSM based modelling in this present investigation as ANOVA based modelling offers lower value of training error i.e. $R M S E$ so $A N O V A$ based model fits better for liquid flow control process modelling.

Table 5. Comparative study based on maximum, minimum and mean of fitness

\begin{tabular}{|c|c|c|c|c|}
\hline Case & Method & Maximum & Minimum & Mean \\
\hline \multirow{3}{*}{ RSM } & Roulette Wheel & $4.7 \mathrm{E}-6$ & $\mathbf{3 . 0 3 E - 6}$ & $3.72 \mathrm{E}-6$ \\
\cline { 2 - 5 } & Tournament & $5.41 \mathrm{E}-5$ & $4.01 \mathrm{E}-6$ & $5.39 \mathrm{E}-5$ \\
\cline { 2 - 5 } & Rank & $7.56 \mathrm{E}-6$ & $3.13 \mathrm{E}-6$ & $4.37 \mathrm{E}-6$ \\
\hline \multirow{4}{*}{ ANOVA } & Roulette Wheel & $5.41 \mathrm{E}-6$ & $\mathbf{5 . 3 9 E}-5$ & $5.40 \mathrm{E}-5$ \\
\cline { 2 - 5 } & Tournament & $5.44 \mathrm{E}-5$ & $\mathbf{5 . 3 9 E - 5}$ & $5.42 \mathrm{E}-5$ \\
\cline { 2 - 5 } & Rank & $5.41 \mathrm{E}-5$ & $\mathbf{5 . 3 9 E - 5}$ & $5.40 \mathrm{E}-5$ \\
\hline
\end{tabular}

\subsection{Reliability Test}

It is desirable that a metaheuristic must always able to reach nearer to the global minima or maxima point as close as possible in every single run. It indicates that the output of the metaheuristic must be reliable for every single run. But due to random initialization and stochastic process of the metaheuristic, the output of the optimization process may vary in different run. However, the variation should be minimal. Therefore, in this subsection, we have tested the reliability of proposed algorithms on the basis of some median, standard deviation and success rate. Comparison with the others existing algorithms have been also made. Median is the measurement of central tendency of the sample or population. The standard deviation is an important statistical parameter which denotes variability or consistency of the data set. Thus, a less value of standard deviation implies a more reliable algorithm. A simulation or program run can be referred as successful if the best-found or minimum fitness value is lesser than or equal to the threshold fitness. This threshold value is decided by some trial and error method. Therefore, the success rate of a metaheuristic is defined as the ration of the number of successful runs (under a particular threshold level) to the total number of runs. Thus, a larger success rate indicates a more reliable optimization technique. In our present problem, a program run is considered to be successful if output best-found fitness value or rmse goes below 1E-3 for modelling of liquid flow control process. Table 6 shows the comparative study based on median \& standard deviation. It can be clearly shown that Tournament selection Genetic Algorithm get smallest values of median (i.e. 9.73E-03) for ANOVA based model; whereas Rank selection of Genetic Algorithm is able to reach smallest values of median (i.e. 3.02E-02) for based model. It is interesting to note that the lowest value of median for ANOVA based model is superior than RSM based model. Tournament selection of a Genetic Algorithm has least standard deviation in the output for Genetic Algorithm based model; whereas Rank selection has lowest standard deviation for RSM based model. However, standard deviations for ANOVA based modelling are better than RSM based model i.e. less fluctuation in result are observed for ANOVA based model or the results are more stable for ANOVA. The reason behind it is that metaheuristic search in lower dimensional space for ANOVA based model ( 5 dimensional search space) compare to the RSM based model (12 dimensional search space). Therefore, RSM based model has greater chance of sticking at local optima. 
Table 6. Comparative study based on median and standard deviation

\begin{tabular}{|c|c|c|c|}
\hline Case & Method & Median & SD \\
\hline \multirow{3}{*}{ RSM } & Roulette Wheel & $5.16 \mathrm{E}-2$ & $3.12 \mathrm{E}-3$ \\
\cline { 2 - 4 } & Tournament & $3.04 \mathrm{E}-2$ & $1.84 \mathrm{E}-3$ \\
\cline { 2 - 4 } & Rank & $3.02 \mathrm{E}-2$ & $1.83 \mathrm{E}-3$ \\
\hline ANOVA & Roulette Wheel & $4.28 \mathrm{E}-2$ & $2.59 \mathrm{E}-3$ \\
\cline { 2 - 4 } & Tournament & $\mathbf{9 . 7 3 E - 3}$ & $\mathbf{6 . 0 4 E}-4$ \\
\cline { 2 - 4 } & Rank & $1.11 \mathrm{E}-2$ & $6.79 \mathrm{E}-4$ \\
\hline
\end{tabular}

\subsection{Computational Efficiency Test}

Besides the previous tests, the computational time is also a major factor for evaluating the efficiency of a metaheuristic. For this purpose, we have observed average execution time taken by each algorithm for each of the problems of liquid flow control process which in turn denotes the computational efficiency of the algorithm. Table 7 shows a comparative study based on average execution time. It has been observed that Rank selection Genetic Algorithm required least computational time for ANOVA based modelling. In case of RSM based modelling, computational efficiency is also best for Rank selection. It is fascinating to observe that ANOVA based modelling required more computational time than RSM based model though the dimension of search space is larger for RSM based model of liquid flow control process. It is due to the fact RSM used polynomial equation for modelling whereas ANOVA used nonlinear power function for the modelling which require more time for calculation.

Table 7. Comparative study based on computational time

\begin{tabular}{|c|c|c|}
\hline Case & Method & Average Computational Time \\
\hline \multirow{4}{*}{ ANOVA } & Roulette Wheel & 89.1393 \\
\cline { 2 - 3 } & Tournament & 138.7695 \\
\cline { 2 - 3 } & Rank & $\mathbf{8 6 . 2 1 1 2}$ \\
\hline \multirow{3}{*}{ RSM based modeling } & Roulette Wheel & 67.4988 \\
\cline { 2 - 3 } & Tournament & 119.0111 \\
\cline { 2 - 3 } & Rank & $\mathbf{6 5 . 0 1 4 8}$ \\
\hline
\end{tabular}

\subsection{Convergence Test}

The above mentioned result and comparisons cannot completely illustrate the performance and the efficiency of the proposed optimization techniques. Therefore, a convergence test has been conducted on liquid flow control process modelling and where we observed the change of best-found fitness values along with the iteration number. So, convergence speeds of the proposed algorithms have been observed to see how the best-found fitness values decrease with the iteration number. For this purpose, we have chosen the output corresponding to the run where we found minimum or best fitness (RMSE) amongst all 20 times run and observe the fitness value at each iteration index. Then, we plot them for all algorithms for RSM and ANOVA based modelling which are shown in following Figure 3 and Figure 4 respectively. In the figures, fitness values are shown up to 1000 iterations only for better clarity and understanding. It can be observed that among the proposed algorithms, Rank selection Genetic Algorithm converges slower compare to the other optimization techniques for AONVA based modelling \& Roulette wheel converge slowly in RSM based GA model.

\subsection{Accuracy Test}

Next, the accuracy test has been conducted to observe liquid flow rate prediction capability under different experimental conditions i.e. sensor output voltage and pipe diameter. Two indexes respectively named as individual absolute error (IAE)\& relative error (RE) and respectively defined as Eqs. 5 and Eqs. 6 are adopted to indicate the 
Table 8. Comparative study based on computational time

\begin{tabular}{|c|c|c|}
\hline Case & Method & Average Computational Time \\
\hline \multirow{4}{*}{ ANOVA } & Roulette Wheel & 89.1393 \\
\cline { 2 - 3 } & Tournament & 138.7695 \\
\cline { 2 - 3 } & Rank & $\mathbf{8 6 . 2 1 1 2}$ \\
\hline RSM based modeling & Roulette Wheel & 67.4988 \\
\cline { 2 - 3 } & Tournament & 119.0111 \\
\cline { 2 - 3 } & Rank & $\mathbf{6 5 . 0 1 4 8}$ \\
\hline
\end{tabular}

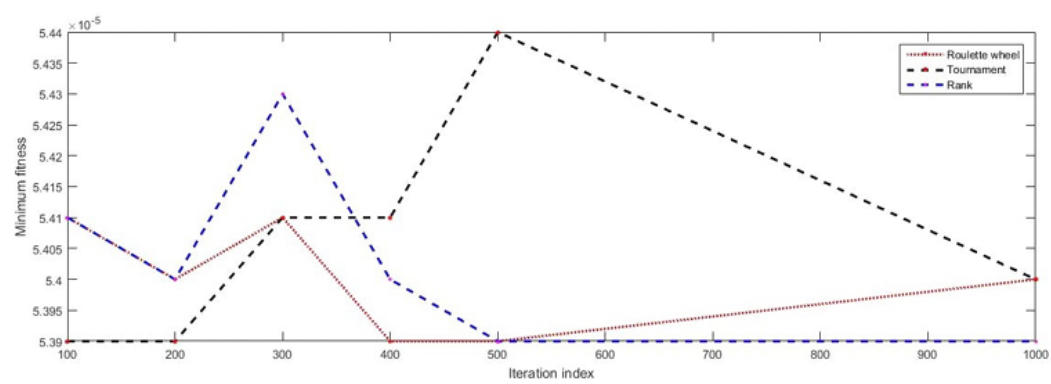

Figure 3. Minimum fitness for ANOVA based modelling of liquid flow process

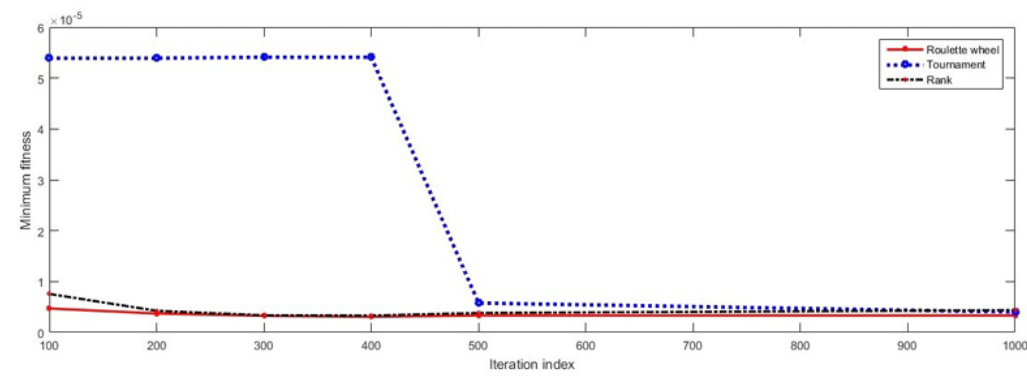

Figure 4. Minimum fitness for RSM based modelling of liquid flow process

error values between the experimental and the simulated current data.

$$
\begin{gathered}
I A E=\left|F_{\text {measured }}-F_{\text {calculated }}\right| \\
R E=\frac{\left|F_{\text {measured }}-F_{\text {calculated }}\right|}{F_{\text {measured }}}
\end{gathered}
$$

Moreover, Total Absolute Error (TAE) can be defined in Eqs. 7:

$$
T A E=\sum_{i=1}^{n} I A E_{i}
$$

where $n$ is the number of measurements in the experimental dataset, $F_{\text {measured }}$ is the experimental value of liquid flow rate and $F_{\text {calculated }}$ is the estimated value of liquid flow rate for a pipe diameter and sensor output voltage. However, to calculate or estimate the values of liquid flow rate of liquid flow control process at different experimental conditions, the best output case of metaheuristics has been considered where output i.e.RMSE is smallest among all different runs. The coefficient of the non linear models is obtained from ANOVA (by using 
EXCEL 2007)\& Response Surface methodology (MINITAB 18.1) shown in Table 9, Table 10 \& Table 11. After knowing the extreme boundary value of the co efficient of a non linear equation from Table10 and Table 11 we get the optimum co efficient of the equation $4 \& 6$ by run the matlab code of Genetic Algorithm. Table 9 shows the statistical parameter of analysis of variance (ANOVA)conduct on response such as liquid flow rate. As the $\mathrm{F}$ value is greater than 3.55 so all the factors are significantly affects response i.e flowrate.

Table 9. Statistical parameter for ANOVA in liquid flow process

\begin{tabular}{|c|c|c|c|c|c|}
\hline & df & SS & MS & F & Significance F \\
\hline Regression & 4 & 34.11307 & 8.528267 & 39.13699 & $6.83768 \mathrm{E}-20$ \\
\hline Residual & 103 & 22.44454 & 0.217908 & & \\
\hline Total & 107 & 56.5576 & & & \\
\hline
\end{tabular}

Table 10. co-efficient of non linear model in ANOVA from EXCEL

\begin{tabular}{|c|c|c|c|c|c|c|}
\hline & Coefficient & Standard Error & t stat & P-value & lower 95\% & lower 95\% \\
\hline Intercept & -6.671000268 & 11.32394126 & -0.58910587 & 0.557080114 & -29.12936633 & 15.78736579 \\
\hline X Variable 1 & 14.20497908 & 1.153586664 & 12.31375112 & $5.76996 \mathrm{E}-22$ & 11.91711208 & 16.49284609 \\
\hline X Variable 2 & -4.318702563 & 0.457142353 & -9.44717228 & $1.25474 \mathrm{E}-15$ & -5.225336576 & -3.41206855 \\
\hline X Variable 3 & -12940439 & 22.4448475 & -0.5765439 & 0.56550543 & -57.454499 & 31.573620 \\
\hline X Variable 4 & 2.9400129 & 2.6535419 & 1.1079579 & 0.270460543 & -2.23226614 & 8.20268726 \\
\hline
\end{tabular}

Table 11. Co efficient of the non linear model Response Surface Methodology from Minitab

\begin{tabular}{|c|c|c|c|c|c|}
\hline Term & Coeff & SE Coeff & T-Value & P-Value & VIF \\
\hline Constant & 0.007469 & 0.000168 & 44.53 & 0.000 & \\
\hline$E$ & 0.01188 & 0.000590 & 20.14 & 0.000 & 14.10 \\
\hline$D$ & -0.005174 & 0.000309 & -16.73 & 0.000 & 12.80 \\
\hline$k$ & 0.00014 & 0.00186 & 0.08 & 0.940 & 473.84 \\
\hline$n$ & 0.00245 & 0.00179 & 1.37 & 0.175 & 434.50 \\
\hline$E^{2}$ & -0.00089 & 0.00114 & -0.78 & 0.438 & 16.12 \\
\hline$D^{2}$ & 0.001189 & 0.000308 & 3.86 & 0.000 & 4.24 \\
\hline$E * D$ & -0.00229 & 0.00104 & -2.21 & 0.029 & 26.18 \\
\hline$E * k$ & -0.00456 & 0.00589 & -0.77 & 0.441 & 1036.52 \\
\hline$E * n$ & -0.00541 & 0.00597 & -0.91 & 0.367 & 1025.08 \\
\hline$D * k$ & -0.00590 & 0.00315 & -1.87 & 0.065 & 921.85 \\
\hline$D * n$ & -0.00561 & 0.00320 & -1.75 & 0.083 & 927.37 \\
\hline
\end{tabular}

Table 12. Co efficient of the different non linear model Response Surface Methodology from Matlab

\begin{tabular}{|c|c|c|c|c|c|c|c|c|c|c|c|c|}
\hline Method & $\beta_{0}$ & $\beta_{1}$ & $\beta_{2}$ & $\beta_{3}$ & $\beta_{4}$ & $\beta_{11}$ & $\beta_{22}$ & $\beta_{12}$ & $\beta_{13}$ & $\beta_{14}$ & $\beta_{23}$ & $\beta_{24}$ \\
\hline Roulette Wheel & -0.014 & 0.0925 & -0.066 & -0.023 & 0 & 0 & -0.100 & 0.100 & 0.064 & $6.2 \mathrm{E}-4$ & 0.0587 & 0.0191 \\
\hline Tournament & -0.007 & 0.100 & -0.025 & -0.029 & 0 & 0 & 0.0217 & 0.0998 & 0.018 & 0.009 & -0.024 & 0.0205 \\
\hline Rank & -0.025 & 0.070 & -0.027 & $4.1 \mathrm{E}-4$ & 0 & 0 & -0.099 & -0.012 & 0.100 & -0.014 & -0.021 & 0.0586 \\
\hline
\end{tabular}

table 13 From Table 10 we found the parameter co efficient of a liquid flow control process .Intercept we get the value of constant value $\mathrm{k}$ in standard ANOVA equation while $\mathrm{X}$ variable $1,2,3$ \& 4 we get the non optimized co efficient of 4 input independent variables sensor output, pipe diameter, liquid conductivity \& liquid viscosity. Table 12 and Table 13 describe the different best optimal parameters values for RSM and ANOVA based 
Table 13. Co efficient of the different non linear model ANOVA from Matlab

\begin{tabular}{|c|c|c|c|c|c|}
\hline Method & $\mu_{1}$ & $\mu_{2}$ & $\mu_{3}$ & $\mu_{4}$ & $\mu_{5}$ \\
\hline Roulette Wheel & 0.0058 & 0.1000 & 0.0950 & 0.100 & -0.100 \\
\hline Tournament & 0.0038 & 0.100 & -0.0149 & 0.0943 & -0.1000 \\
\hline Rank & 0.0058 & 0.1000 & 0.0966 & 0.1000 & -0.1000 \\
\hline
\end{tabular}

modelling of liquid flow control process respectively obtain from the matlab code where presumed variable co efficient are taken from the Table $10 \&$ Table 11.Using these parameters, values of liquid flow rate of the liquid flow $\&$ level measurement $\&$ control unit can be estimated for different cases or conditions. Table 14 describes a comparative study based on total absolute error. From Table 14, it can be clearly seen that Tournament selected Genetic Algorithm has the best performances in term of total absolute error for RSM based modelling as well as ANOVA based modelling. However, total absolute error for RSM based modelling (using all metaheuristics) is much lower than ANOVA based model. It signifies that the RSM based method can predict the values of liquid flow rate more accurately for all cases compare to the ANOVA based model.

Table 14. Comparative study based on total absolute error

\begin{tabular}{|c|c|c|}
\hline Case & Method & TAE \\
\hline \multirow{4}{*}{ ANOVA } & Roulette Wheel & 0.0423 \\
\cline { 2 - 3 } & Tournament & $\mathbf{0 . 0 2 5 0}$ \\
\cline { 2 - 3 } & Rank & 0.0251 \\
\hline RSM based modeling & Roulette Wheel & 0.0421 \\
\cline { 2 - 3 } & Tournament & $\mathbf{0 . 0 0 8 0}$ \\
\cline { 2 - 3 } & Rank & 0.0093 \\
\hline
\end{tabular}

Figures 5 and Figure 6 show the relative errors vs. different liquid flow rate measurement instances for RSM and ANOVA based modelling respectively. It can be clearly seen that the proposed RSM based model has less relative error compare to the ANOVA based model.

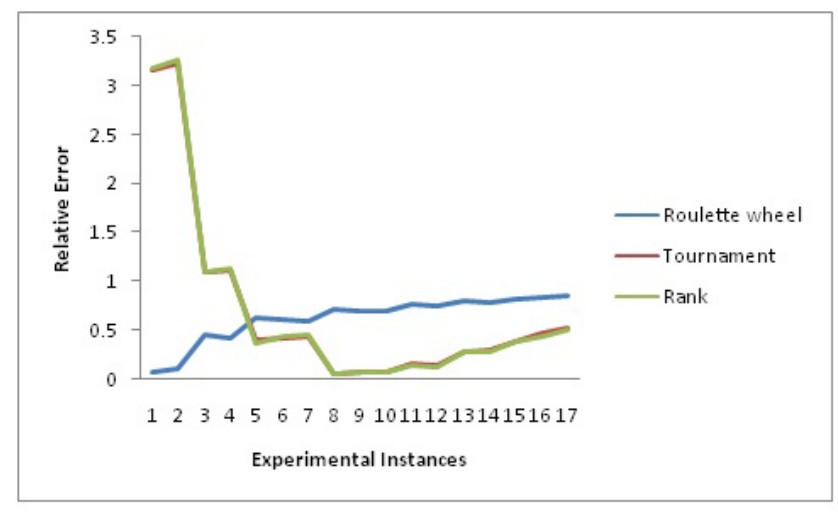

Figure 5. Relative errors for GA-ANOVA based modelling of liquid flow control process

However, Figure 7 and Figure 8 show the experimental data and estimated liquid flow rate of liquid flow control process for RSM and ANOVA based model respectively using all proposed algorithms. Here, the liquid flow rate are calculated using best output or optimal model parameters (where RMSE or fitness is smallest amongst all program run) which are shown in Table 13 and Table 12 respectively. From Figure $9 \&$ Figure 10 it can be observed that the proposed Tournament \& Rank selected Genetic Algorithm can predict the liquid flow rate with greater accuracy or satisfactorily for the cases of RSM based model. Roulette Wheel has significantly more deviation shown in 


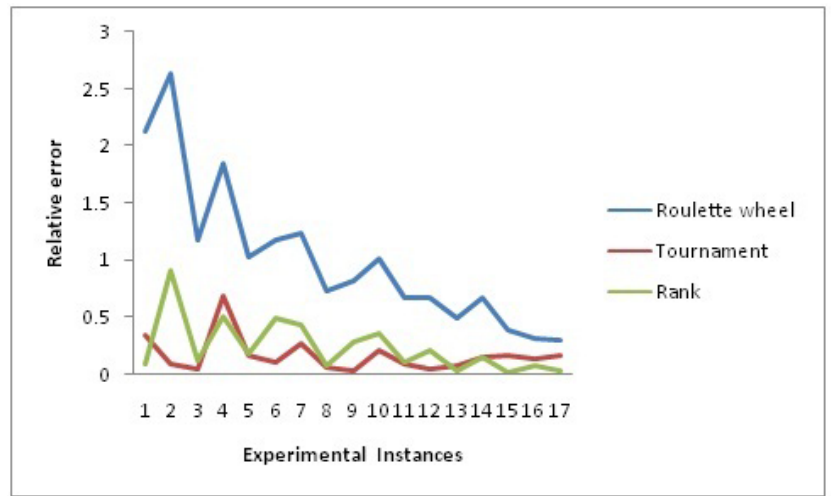

Figure 6. Relative errors for GA-RSM based modelling of liquid flow control process

Figure 8. On the other hand, all proposed improved versions of Genetic Algorithm except Roulette Wheel perform same way for the prediction of liquid flow rate using ANOVA base modelling. It can be clearly seen that RSM based modelling has superior prediction capability compare to the ANOVA based model. Moreover, the prediction capability helps to validate the efficiency of the proposed algorithms.

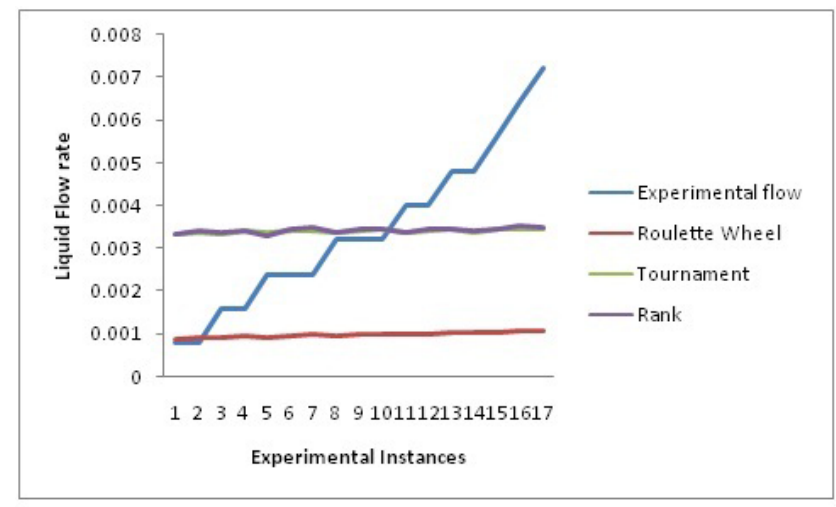

Figure 7. Comparison of the characteristics of the experimental data and estimated liquid flowrate using ANOVA based model

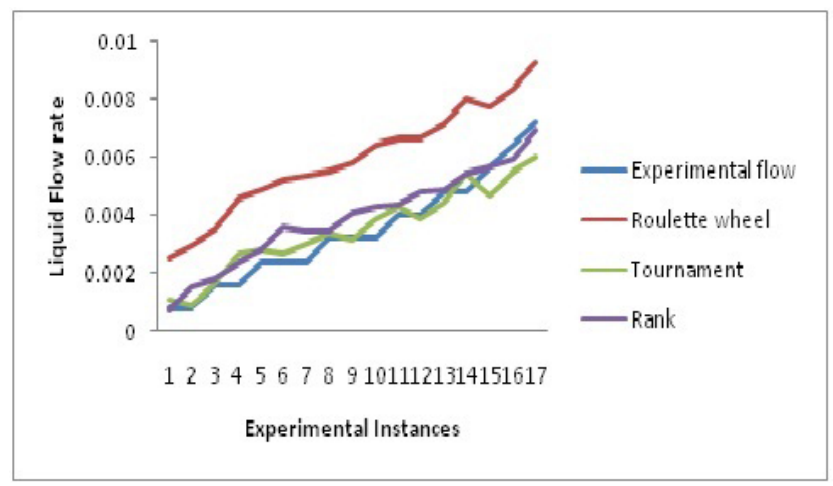

Figure 8. Comparison of the characteristics of the experimental data and estimated liquid flowrate using RSM based model 


\subsection{Overall Efficiency}

Now we summarize the performance of algorithms based on above mentioned evaluation criterions and compare among themselves. Therefore, we assigned a performance score against each algorithm for each of the criterions. The value of this score is calculated as the ratio of number of cases (functions) where an algorithm achieves best result (criterion) to the total number of cases (i.e. RSM and ANOVA based modelling of liquid flow control process). Table 15 shows the comparative study based on these scores to evaluate overall efficiency of the proposed algorithms. The maximum score obtained by any algorithm for each of the performance criterions is highlighted in bold letter.

It is clearly noticed from following table that Rank selected Genetic Algorithm performed best or better than others GA selected Algorithm for all conditions except convergence speed and Total Absolute error \& relative error. Roulette wheel is better for finding the best fitness. Another important fact is observed that Roulette wheel is better for ANOVA modelling, whereas Rank selected GA is very much suitable for the RSM based modelling of liquid flow control process. Moreover Rank selected GA is found to be best efficient algorithm with respect to RMSE, Median ,standard deviation \& execution time .On the other hand, Roulette wheel has better stability in output.

In summary, all the proposed algorithms have satisfactory performance for the modelling of liquid flow control process. However, some of them performed slightly better at certain performance criterion and others are also performed better based on others criterions. Overall, Rank selected GA achieved highest performance score which indicate that it is most efficient and suitable optimization technique among the improved version of GA.

Table 15. Comparative study based on score of different performance criterions

\begin{tabular}{|c|c|c|c|c|c|c|c|c|c|c|}
\hline $\begin{array}{l}\frac{n}{0} \\
\stackrel{\Xi}{0} \\
\sum^{e}\end{array}$ & 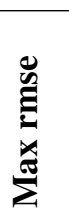 & $\stackrel{\Xi}{\Xi}$ & 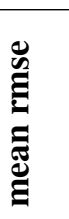 & 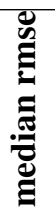 & $\stackrel{\dot{\phi}}{\dot{\omega}}$ & $\stackrel{\mathscr{\Xi}}{\Xi}$ & 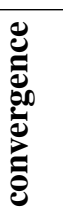 & $\sum_{5}^{5}$ & 空 & $\stackrel{\text { ț }}{0}$ \\
\hline ANOVA+Roulette & 2 & 2 & 2 & 0 & 0 & 1 & 1.5 & 0 & 2 & 10.5 \\
\hline $\begin{array}{l}\text { ANOVA +Tourna- } \\
\text { ment }\end{array}$ & 0 & 0 & 0 & 2 & 2 & 0 & 1.5 & 2 & 1 & 8.5 \\
\hline ANOVA+Rank & 1 & 1 & 1 & 1 & 1 & 2 & 0 & 1 & 0 & 8 \\
\hline RSM +Roulette & 1.5 & 1 & 1.5 & 0 & 0 & 1 & 2 & 0 & 0 & 7 \\
\hline $\begin{array}{l}\text { RSM } \\
\text { +Tournament }\end{array}$ & 0 & 1 & 0 & 1 & 1 & 0 & 0 & 2 & 2 & 7 \\
\hline RSM+Rank & 1.5 & 1 & 1.5 & 2 & 2 & 2 & 1 & 1 & 1 & 13 \\
\hline
\end{tabular}

\section{Conclusions}

Modelling of liquid flow control in a process industry is an interesting task for the researchers. Generally, liquid flow rate of Liquid Flow \& Level Measurement depends upon the voltage output of sensor (Anemometer type flow sensor), diameter of the pipe, liquid(water) viscosity and conductivity.Initially,134 number of measurements (i.e. liquid flow rate) have been observed from laboratory at different experimental conditions (i.e. for different values of pipe diameter and sensor voltage ,flow rate, liquid conductivity, viscosity ).Among this data sets 117 number of datasets used for generating the objective function by using ANOVA \& rest 17 number of datasets is used for testing purpose. In this study, our aim is to model the liquid flow control process so that we can find a relationship between liquid flow rate, pipe diameter, sensor output voltage, water viscosity \& conductivity (by keeping the water density at constant level).For this modelling purpose, we have used Response Surface Methodology (RSM) 


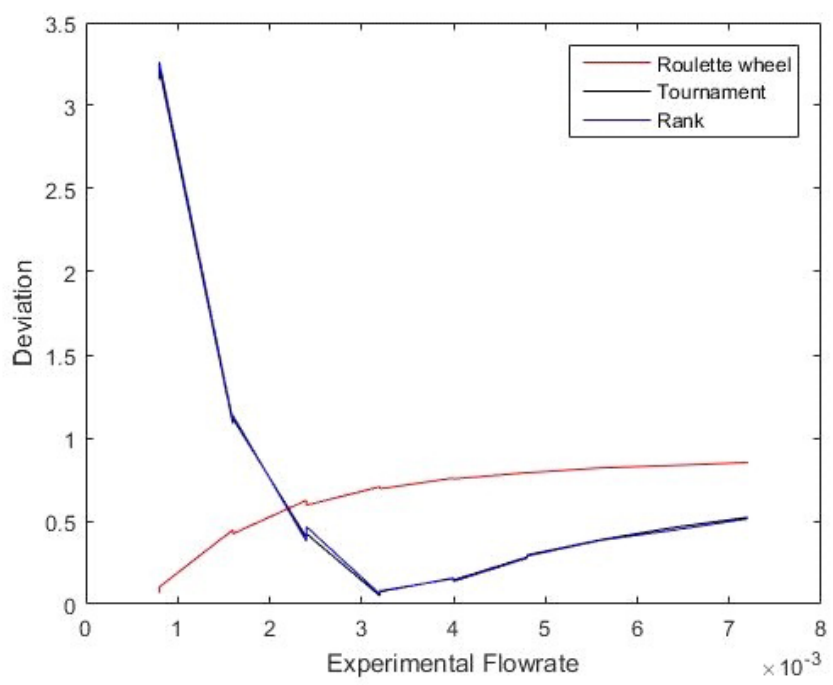

Figure 9. Deviation vs. Experimental flowrate in GA-ANOVA

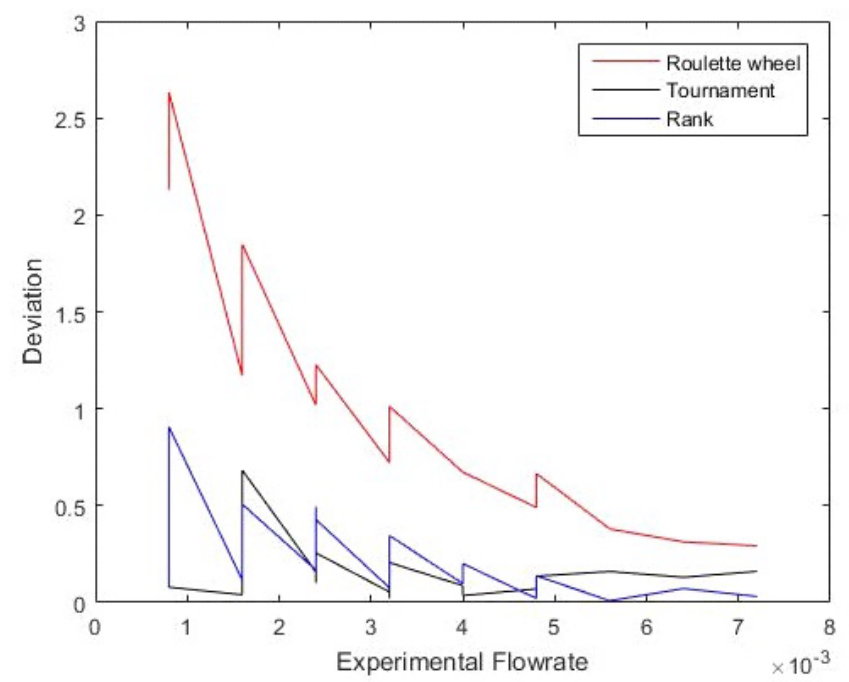

Figure 10. Deviation vs. Experimental flowrate in GA-RSM

and Analysis of Variance (ANOVA) as non-linear models to establish the relationship between variables of liquid flow control process. Now, finding out the suitable RSM and ANOVA based model is essentially a non-linear optimization problem. We need to find out the optimal values of the coefficient of the models using some suitable metaheuristic so that estimated liquid flow rate fit best with the experimental results. For this purpose, we have proposed three new different pattern selection(Roulette wheel, Tournament \& Rank) Genetic Algorithm version and observed their efficiency for the modelling of liquid flow control process.

Numerical simulations are performed and the statistical analysis of the results is also given. All the results indicate that the performances of the proposed Rank selected Genetic Algorithm outperformed the others for the most of the cases of modelling for liquid flow control process for RSM modelling, but one of the major disadvantages of Rank selected GA is its high magnitude of total absolute error \& relative. On the other hand, 
Tournament \& Roulette wheel is better for finding the best fitness. Moreover, Rank selected GA is found to be best efficient algorithm with respect to RMSE and computational time. However, all algorithms can predict the liquid flow rate with satisfactory accuracy.

It is also found that RSM based model fits better than the ANOVA based model to characterize the liquid flow control process as rmse, $I A E$ and $R E$ are less for RSM based model. Moreover, average computational time for RSM based model is also lesser than ANOVA based model. However, due to requirement of higher dimensional search for RSM based model, the stability and success rate is slightly inferior than ANOVA based model. It has been found that Roulette wheel is better for ANOVA modelling, whereas Rank selected GA is very much suitable for the RSM based modelling.

More detailed and accurate modelling of the liquid flow control process (including liquid density as a input variable) is the future aspect of this work. Moreover, further tunings of the metaheuristics are necessary to achieve more efficiency, accuracy, convergence speed, stability and success rate.

\section{REFERENCES}

1. P. Dutta, S. Mandal, and A. Kumar, Comparative study :FPA based response surface methodlogy and ANOVA for the parameter Optimization in Process Control, Advances in Modelling and analysis C, vol. 73, no. 1, pp. 23-27, 2018.

2. P. Dutta, S. Mandal and A. Kumar, Application of FPA and ANOVA in the Optimization of Liquid Flow Control Process, Review of Computer Engineering, vol. 5, no. 1, pp. 7-11, 2018.

3. S. C. Bera, B. Chakraborty, and D. N.Kole, Study of a Modified Anemometer Type Flow Meter, Sensors \& Transducers Journal, vol. 83, no. 9, pp. 1521-1526, 2007.

4. S. C. Bera, and M. Samik Study of a simple linearization technique of a p-n junction typeanemometer flow sensor, IEEE Transaction Instrumentation and Measurement, vol. 61, no. 9, pp. 545-552, 2012.

5. S. C. Bera, and J. K. Roy, An approach to the design and fabrication of a micro processor based flow meter using resistance and semiconductor probe, IETE Technical Review, vol. 18, no. 5, pp. 355-360, 2001.

6. K. V. Santosh, and K. V. Roy, An Intelligent Flow Measurement Technique using Ultrasonic Flow Meter with Optimized Neural Network, International Journal of Control and Automation, vol. 5, no. 4, pp. 185-196, 2012.

7. P. Dutta, and A. Kumar, Intelligent calibration technique using optimized fuzzy logic controller forultrasonic flow sensor, Mathematical Modelling of Engineering Problems , vol. 4,no. 2, pp. 91-94, 2017.

8. P. Dutta, and A. Kumar, Design an intelligent flow measurement technique by optimized fuzzy logic controller, Journal Europen des Systmes Automatiss, vol. 51, pp. 89-107, 2018.

9. P. Dutta, and A. Kumar, Study of Optimizated NN model for Liquid Flow sensor Based on Different Parameters, International conference on Materials, Applied Physics \& Engineering, 2018.

10. P. Dutta, and A. Kumar, Flow sensor Analogue:Realtime Prediction Analysis using SVM \& KNN, 1st International conference on Emerging trends in Engineering \& science (ETES) on 23rd-24th March, 2018.

11. P. Dutta, and A. Kumar, Design an intelligent calibration technique using optimized GA-ANN for liquid flow control system, Journal Europen des Systmes Automatiss, vol. 50, no. 4-6, pp. 449-470, 2017.

12. P. Dutta, and A. Kumar, Application of an ANFIS model to Optimize the Liquid Flow Rate of a Process Control System, Chemical Engineering Transactions, vol. 71, pp. 991-996, 2018.

13. P. Dutta, and A. Kumar, Modeling and Optimization of a Liquid Flow Process using an Artificial Neural Network-Based Flower Pollination Algorithm, journal of intelligence system, https://doi.org/10.1515/jisys-2018-0206, 2018.

14. P. Dutta,S. Mandal, and A. Kumar, Modeling of Liquid Flow Control Process Using Improved Versions of Elephant Swarm Water Search Algorithm, SN Applied Sciences, SN Appl. Sci. (2019) 1: 886. https://doi.org/10.1007/s42452-019-0914-5, 2019.

15. L. Bianchi, A survey on metaheuristics for stochastic combinatorial optimization, Natural computing: an international Journal, vol. 8, no. 2, pp. 239-287, 2009.

16. R. C. Eberhart, Y. H. Shi, Comparing inertia weights and constriction factors in particle swarm optimization, Proceeding of IEEE Congress on Evolutionary Computation, pp. 84-88, 2000.

17. X. S. Yang, A New Metaheuristic Bat-Inspired Algorithm, Nature Inspired Cooperative Strategies for Optimization (NISCO 2010), vol. 284, pp. 65-74, 2010.

18. X. S. Yang, and S. Deb, Engineering Optimisation by Cuckoo Search, Int. J. Mathematical Modelling and Numerical Optimisation, vol. 1, no. 4, pp. 330-343, 2010.

19. X. S. Yang, Flower pollination algorithm for global optimization, Proceeding of Unconventional Computation and Natural Computation 2012, Lecture Notes in Computer Science, vol. 7445, pp. 240-249, 2012.

20. X. S. Yang, Firefly algorithm, stochastic test functions and design optimization, International Journal of Bio-Inspired Computation, vol. 2, pp. 78-84, 2010.

21. M. Dorigo, V. Maniezzo, and A. Colorni, Ant System: Optimization by a colony of cooperating agents, IEEE Transactions on Systems, Man, and Cybernetics C Part B, vol. 26, no. 1, pp. 29-41, 1996.

22. D. Karaboga, and B. Basturk, A powerful and efficient algorithm for numerical function optimization:artificial bee colony (ABC) algorithm Journal of Global Optimization, vol. 39, no. 3, pp. 459-471, 2007.

23. S. Mirjalili, S. M. Mirjalili, and A. Lewis, Grey wolf optimizer, Advances in engineering software, vol. 69, pp. 46-61, 2014.

24. S. Mirjalili, and Lewis, The whale optimization algorithm, Advances in Engineering Software, vol. 95, pp. 51-67, 2016. 
25. D. H. Wolpert, and W. G. Macready, No Free Lunch Theorems for Optimization, IEEE Transactionson Evolutionary Computation, vol. 1 , no. 1, pp. 67-82, 1997.

26. M. Montazeri, A. Poursamad, and B. Ghalichi, Application of genetic algorithm for optimization of control strategy in parallel hybrid electric vehicles, Journal of the Franklin Institute, Vol. 343, no. 4C5, pp. 420-435, 2006.

27. M. Loomans, and X. Visser, Application of the genetic algorithm for optimisation of large solar hot water systems, Solar Energy, Vol. 72, no. 5, pp. 427-439, 2002.

28. M. Lin, Application of Optimized Genetic Algorithm in Building Energy-Saving Optimization Control, Lecture Notes in Real-Time Intelligent Systems, pp. 182-188, 2017.

29. L. M. Fernandes, I.N. Figueiredo, J. J. Judice, L.A. Costa, and P.N. Oliveira, Application of Genetic Algorithms to plate optimization, Proceedings of the Fourth Congress on Computational Mechanics,Computational mechanics, Barcelona, Spain, 1998.

30. A. A. Freitas, A survey of Evolutionary Algorithms for Data Mining and Knowledge Discovery, Advances in Evolutionary Computation, Ser. A, vol. 92, pp. 103-118, 2002.

31. M. Pei, E. D. Goodman, and W. F. Punch, Pattern Discovery from Data Using Genetic Algorithms, Proceeding of 1st Pacific-Asia Conference Knowledge Discovery \& Data Mining (PAKDD-97), 1997.

32. P. L. Schoonover, W. A. Crossley, and W. A. Heister, Application of Genetic Algorithm to the optimization of hybrid Rockets, Journal of Space craft and Rockets, vol. 37, no. 5, pp. 991-996, 2000.

33. M. A. Bezerra, R. E. santelli, E. P. Oliveira, L. S. Villar, L. S. Escaleria, response surface methodology $(R S M)$ as a Tool for Optimization in analytical Chemistry, Talanta, vol. 76, pp. 965-977, 2008.

34. S. A. Glantz, B. K. Slinker, and T. B. Neilands, Primer of applied regression \& analysis of variance, Mcgraw-Hill Medical publishing Division, 2016.

35. H. J. Keselman, C. J. Huberty, L. M. Lix, R. A. cribbie, Statistical practices of educational researches:analysis of their ANOVA, MANOVA, and ANOCOVA analysis, Review in Education \& Research, vol. 68, pp. 350-386, 1998.

36. Z. Michalewicz, Genetic Algorithms + Data Structures = Evolution Programs, 3rd Ed. Springer-Verlag, 1996.

37. Y. Park, and Song M, genetic algorithm for clustering problems. Genetic Programming 1998, Proceeding of 3rd Annual Conference, pp. 568-575. Morgan Kaufmann, 1998.

38. A. K. Jain, Zongker D, Feature Selection: Evaluation, Application, and Small Sample Performance, IEEE Transaction on Pattern Analysis and Machine Intelligence, vol. 19, no. 2, pp. 153-158, 1997.

39. E. Falkenauer, Genetic Algorithms and Grouping Problems, John Wiley \& Sons, 1998. 